low decision latitude at work and risk for RA was explained by differences in smoking and educational level. However, both low social support and low decision latitude at work associate strongly with known, and here validated, risk factors for RA (smoking, obesity, no university degree) with similar pattern among both cases and controls.

Acknowledgement: The EIRA study group

Disclosure of Interests: Louise Hedenstierna: None declared, Christina $\mathrm{H}$. Opava: None declared, Sofia Ernestam: None declared, Johan Askling Grant/research support from: Karolinska Institutet (JA) has or has had research agreements with the following pharmaceutical companies, mainly in the context of the ATRIS national safety monitoring programme for rheumatology biologicals: Abbvie, BMS, MSD, Eli Lilly, Pfizer, Roche, Samsung Bioepis, and UCB., Consultant for: Karolinska Institutet has received remuneration for JA participating in ad boards arranged by Lilly, Novartis, and Pfizer., Lars Klareskog Grant/research support from: Yes, but not for the presented study., Lars Alfredsson: None declared, Saedis Saevarsdottir Employee of: Part-time employee at deCODE Genetics/ Amgen Inc, working on genetic research unrelated to this project. DOI: 10.1136/annrheumdis-2019-eular.6088

\section{SAT0047 PRIMARY LYMPHOID TISSUE FROM RHEUMATOID ARTHRITIS PATIENTS HARBOUR CITRULLINE SPECIFIC PLASMA CELLS}

Radha Thyagarajan ${ }^{1}$, Aase Hensvold ${ }^{1,2}$, Lena Israelsson ${ }^{1}$, Johanna Steen ${ }^{1}$ Heidi Wähämaa ${ }^{1}$, Annika van Vollenhoven ${ }^{1}$, Khaled Amara ${ }^{1}$, Anca Catrina ${ }^{1,2}$ Vivianne Malmström ${ }^{1}$, Caroline Grönwall ${ }^{1} .{ }^{1}$ Rheumatology unit Karolinska University Hospital and Karolinska Institutet, Stockholm, Sweden; ${ }^{2}$ Center for Rheumatology, Academic Specialist Center, Stockholm Health Services, Stockholm, Sweden

Background: Anti-citrullinated protein antibodies (ACPA) are specific markers with pathological effects in rheumatoid arthritis (RA). ACPA-specific B-cells have been identified in synovial joint fluid and peripheral blood. An increased concentration of ACPA is found in synovial fluid and lung tissue compared to blood suggest local production, partly confirmed by detection ACPA synovial plasma blasts, but primary lymphoid tissue have not previously been examined.

Objectives: In this study we investigated the plasma cell repertoire in the bone marrow and occurrence of autoantibody producing plasma cells. Methods: For this study we developed a method to collected and process bone marrow samples from proximal femur in RA patients undergoing hip joint replacement. Bone marrow samples were processed and mononuclear cells were obtained by Ficoll separation. CD138+ plasma cells were single cell sorted by flow cytometry. Paired heavy and light chains were PCR amplified, sequenced, and analyzed by V-Quest and IgBLAST towards the IMGT database to annotate variable gene usage. To enrich for ACPA, sequences were selected based on high somatic hypermutations number and Fab N-glycosylation sites. Selected sequences were cloned and expressed as IgG in Expi293 cells. Monoclonal antibody reactivity to citrullinated and arginine form of vimentin, enolase, fibrinogen, histone, tenascin C peptides; carbamylated and malondialdehyde-acetaldehyde bovine serum albumin, and tetanus toxoid antigens were examined by ELISA. Included patients were clinical examined and donating peripheral blood samples.

Results: After method development and quality analysis we were able to include five bone marrow samples in this study. The five included RA patients, median age 74 (range 66-82) years, had in median 20 (range 4-44) years of destructive disease, all were RF positive, four were females and anti-CCP2-positive, and all except one were treated with anti-rheumatic drugs.

Overall, from the processed bone marrow, 465 paired heavy-light IgG plasma cell sequences were obtained, in total 368 (range 20-194) from ACPA positive patients and 97 from the ACPA negative patient. We observed statistically significant changes in heavy chain variable gene usage with lower $\mathrm{VH}-1$ and higher $\mathrm{VH}-3$ frequency in sequences from ACPA positive RA patients compared to sequences from the ACPA negative RA patient. We also found statistically significant increase in $\mathrm{VH} N-$ glycosylation in sequences from the ACPA positive RA patients $(22.6 \%$ ACPA positive vs $12.4 \%$ ACPA negative; $p=0.03$ ) but no difference in mutation numbers. From obtained sequences, 34 interesting clones were selected for monoclonal antibody-expression. Among the 34 clones we found two citrulline specific reactive clones (reactivity towards CCP2, citrullinated vimentin and fibrinogen peptides) and one malondialdehyde-acetaldehyde (MAA) reactive clone, originating from three different APCA positive patients with the most longstanding disease. None of these clones had reactivity towards control antigens such as arginine versions of peptides.

Conclusion: Plasma cells producing ACPA are present in primary lymphoid tissue from RA patients. Further studies of difference in characteristics, IgG gene usage and $\mathrm{N}$-glycosylation, between ACPA-positive and ACPA-negative patients are needed.

Disclosure of Interests: Radha Thyagarajan: None declared, Aase Hens vold: None declared, Lena Israelsson: None declared, Johanna Steen: None declared, Heidi Wähämaa: None declared, Annika van Vollenhoven: None declared, Khaled Amara: None declared, Anca Catrina Grant/ research support from: Yes, but not for the presented study., Vivianne Malmström: None declared, Caroline Grönwall: None declared DOI: 10.1136/annrheumdis-2019-eular.5961

\section{SAT0048 1 ANTI-FRACTALKINE MONOCLONAL ANTIBODY AMELIORATE JOINT DESTRUCTION AND SYNOVIUM THROUGH SUPPRESSION OF OSTEOCLAST PRECURSOR MIGRATION AND INDUCTION OF SYNOVIAL CELL DEATH IN COLLAGEN-INDUCED ARTHRITIS MODEL}

Naoto Ishii ${ }^{1}$, Kana Hoshino-Negishi ${ }^{1}$, Masayoshi Ohkuro ${ }^{2}$, Tomoya Nakatani ${ }^{1}$, Wataru Ikeda ${ }^{1}$, Yoshikazu Kuboi ${ }^{1}$, Nobuyuki Yasuda ${ }^{1}$, Toshio Imai ${ }^{1}{ }^{1}$ KAN Research Institute Inc., Kobe, Japan; ${ }^{2}$ EA Pharma Co., Ltd., Kawasaki, Japan

Background: In the Phase 1/2 clinical study, E6011, a novel humanized anti-fractalkine $(F K N)$ mAb demonstrated a promising efficacy in active RA patients who were inadequately controlled by MTX and/or TNF- $\alpha$ inhibitors (NCT 02196558) $)^{1}$.In RA joint tissue, increased expression of fractalkine (FKN) and abundant infiltration of CX3CR1-positive cells were observed $^{2}$. FKN is expressed on endothelial cells and fibroblast-like synoviocytes in synovium and also expressed on osteoblasts. CX3CR1 is expressed on monocytes/macrophages and osteoclast precursors (OCPs). Therefore, FKN-CX3CR1 interaction could play pivotal roles in migration, differentiation and activation of those cells. However, the precise mechanism(s) of FKN-CX3CR1 axis in RA, especially on joint destruction remains to be elucidated.

Objectives: To elucidate the roles of FKN-CX3CR1 axis in cartilage destruction, bone damage and proliferated synovial cells by using antimouse FKN mAb (anti-mFKN mAb).

Methods: For the induction of $\mathrm{ClA}$, mice were immunized with bovine type II collagen. Anti-mFKN mAb was injected twice a week. The clinical arthritis score was monitored, and joint destruction was evaluated by soft $x$-ray and histology. The mRNA expression levels were assessed by quantitative RT-PCR. Blood parameters were measured using ELISA. In in vitro, effect of immobilized FKN on RANK ligand (RANKL)-induced osteoclast differentiation was examined. In in vivo, bone marrow-derived OCPs were fluorescein-labeled and transferred to $\mathrm{ClA}$ mice to evaluate the migration of OCPs into synovium. Inhibitory effect of anti-mFKN mAb, etanercept or tofacitinib against OCP migration was assessed. To examine the effect of anti-mFKN mAb against proliferated synovial cells, propidium iodide $(\mathrm{PI})$ was injected to anti-mFKN mAb-treated CIA mice to detect the synovial cell death.

Results: Anti-mFKN mAb significantly reduced the arthritis and soft x-ray scores in both prophylactic and therapeutic treatment. Anti-mFKN mAb histologically improved synovitis, cartilage destruction and bone damage with marked reduction of osteoclast numbers. Plasma levels of COMP and MMP-3 were also decreased. Interestingly, anti-mFKN mAb significantly suppressed Tnf and II6 mRNA expression in the affected joints. In in vitro, RANKL-induced osteoclast differentiation was enhanced by immobilized FKN, and anti-mFKN mAb suppressed FKN-dependent osteoclas formation. In in vivo, anti-mFKN mAb strongly inhibited the migration of OCPs into the proliferated synovial cells of mice with CIA, whereas etanercept or tofacitinib had no significantly effect. Importantly, synovial cell death was abundantly found in proliferated synovial cells of mice with $\mathrm{CIA}$ after the anti-mFKN mAb treatment ${ }^{3}$.

Conclusion: Anti-mFKN mAb remarkably ameliorated the joint destruction with the marked reduction of osteoclasts by the inhibition of both OCP survival and OCP migration in inflamed joint tissues. In addition, antimFKN mAb immediately induced synovial cell death in the proliferated synovial cells, suggesting the direct inhibitory effect in the synovitis. These results strongly indicate that inhibition of FKN-CX3CR1 axis by a humanized anti-FKN mAb, E6011, is an attractive and affected jointsselective therapeutic strategy for the treatment of both inflammatory synovitis, cartilage destruction and bone damage in RA patients.

\section{REFERENCES}

[1] Tanaka Y, et al., Mod Rheumatol (2018) 28, 58-65 
[2] Nanki T, et al., Arthritis Rheum (2002) 46, 2878-83

[3] Hoshino K, et al., Arthritis Rheumatol (2018) Aug 6 [Epub ahead of print]

Disclosure of Interests: NAOTO ISHII Shareholder of: Eisai Co., Ltd., Employee of: KAN Research Institute, Inc. (subsidiary of Eisai Co., Ltd.), Kana Hoshino-Negishi Shareholder of: Eisai Co., Ltd., Employee of: KAN Research Institute, Inc. (subsidiary of Eisai Co., Ltd.), Masayoshi Ohkuro Employee of: EA Pharma Co., Ltd., Tomoya Nakatani Employee of: KAN Research Institute, Inc. (subsidiary of Eisai Co., Ltd.), Wataru Ikeda Shareholder of: Eisai Co., Ltd., Employee of: KAN Research Institute, Inc. (subsidiary of Eisai Co., Ltd.), Yoshikazu Kuboi Shareholder of: Eisai Co., Ltd., Employee of: KAN Research Institute, Inc. (subsidiary of Eisai Co., Ltd.), Nobuyuki Yasuda Shareholder of: Eisai Co., Ltd., Employee of: KAN Research Institute, Inc. (subsidiary of Eisai Co., Ltd.), Toshio Imai Shareholder of: Eisai Co., Ltd., Employee of: KAN Research Institute, Inc. (subsidiary of Eisai Co., Ltd.)

DOI: 10.1136/annrheumdis-2019-eular.1790

\section{SAT0049 INFECTION WITH CITRULLINATING PORPHYROMONAS GINGIVALIS CAN INDUCE AUTOIMMUNITY TO HUMAN RIBOSOMAL PROTEINS AND TNF ALPHA TREATMENT NONRESPONSE}

Madeleine Jenning ${ }^{1}$, Bianka Marklein ${ }^{1}$, Ute Nonhoff $^{2}$, Zoltan Konthur ${ }^{2}$, Gerd Rüdiger Burmester ${ }^{1}$, Karl Skriner ${ }^{1} .{ }^{1}$ Charité University Medicine, Department of Rheumatology and Clinical Immunology, Berlin, Germany, ${ }^{2}$ Max Plank Institute, Max-Planck-Institut für Kolloid- und Grenzflächenforschung, Potsdam, Germany

Background: Porphyromonas gingivalis ( $\mathrm{P}$ g.) is involved in triggering self-reactive immune responses when cirtullinating bacterial or human proteins. However, first evidence to link anti-ribosomal $T$ and $B$ cells responses to rheumatoid arthritis (RA) has been published but the mechanism and its influence on therapy is not clear (1). Infection based autoimmunity induced by citrullination of human proteins with $P$ g. peptidyl arginine deiminase from RA patient (RA-PPAD) and crossreactivity binding induced by $\mathrm{P} g$. was investigated using patient sera, affinity purified RA patient antibodies and monoclonal antibodies to cit-RA-PPAD.

Objectives: Antibodies to RA-PPAD isolated from an RA patients (RAPPAD) was first time linked to target specific citrullinated ribosomal proteins and therapy.

Methods: Screening of RA sera was conducted on 37.830 unique human proteins on protein marcoarrays (http://www.engine-gmbh.de) with $30 \mathrm{RA}$ sera. The autoantibody response to 840 different proteins was recorded and bioinformatically evaluated. Protein arrays were citrullinated with human peptidyl arginine deiminase PAD 2, 4, rabbit PAD and RA-PPAD from P.g. Sera and affinity purified antibodies were used to detect reactivity to 840 autoantigens and 15aa CCP peptide form RA-PPAD. Sera from TBA treated sera anti-TNF (adalimumab, etanercept, certolizumab) treatment were tested with the cit-PPAD-peptide of 15aa (CPP).

Results: A human protein macroarray consisting of 840 indentified autoantigens from RA patients was modified by human PAD2 and PAD4, rabbit $P A D$, and RA-PPAD form $P$ g. Using cit specific monoclonal antibodies we identified the ribosomal proteins (RP), RPL18a, RPS27a, modified by PAD2, RPL18a and MRPS11 modifies by PAD4, and RPL7L1 modified by rabbit PAD specifically targeted. In addition 6 RA patient sera and 3 osteoarthritis $(\mathrm{OA})$ control sera were used to identify the citrullinated RAPPAD specific modified autoantigens not targeted when modified by human PAD2 or PAD4 or rabbit PAD. We identified the RA-PPAD citrullinated ribosomal Proteins RPL3, RPL21, RPS24, RPL9, RPL15, RPS24, RPS3a, MRPL28 specifically targeted by RA patients. This identifies ribosomal proteins as major specific RA-PPAD citrullination targets. Moreover, affinity purified antibodies bound to native and citrullinated RA-PPAD from 6 RA patient sera and 3 OA patient sera were tested for crossreactvity on citrullinated human proteinarray. Antibodies to citrullinated ribosomal proteins MRPS11, RPL21, RPS3a, RPL18a, RPS27a, MRPL28 were detected in the RA group but not in the OA control group. High antibody titre against the cit-PPAD-peptide of 15aa (CPP) derived from the autocitrullination site (R63) of RA-PPAD correlates with TNFo-inhibitor (TBA) non-response $(n=61)$. DMARD patients refractory to different treatment regimes $(n=61)$, receiving anti-TNF (adalimumab, etanercept, certolizumab), do not respond when maintaining high $\alpha$-CPP lgG level.

Conclusion: Failure of Porphyromonas gingivalis clearance in RA patients leads to infection induced enzymatic mimicry based autoreactivity targeting evolutionary conserved human ribosomal proteins. Autoimmunity to ubiquitous self-antigens may trigger localized tissue damage in RA.TBA nonresponse leads to the suggestion to clear Porphyromonas gingivalis infection before $\alpha$-TNF treatment.

\section{REFERENCE}

[1] :Ito, Y. et al. Detection of $T$ cell responses to a ubiquitous cellular protein in autoimmune disease. Science 346, 363-368 (2014)

Disclosure of Interests: Madeleine Jenning: None declared, Bianka Marklein: None declared, Ute Nonhoff: None declared, Zoltan Konthur: None declared, Gerd Rüdiger Burmester Consultant for: Roche, Sanofi-Genzyme, Speakers bureau: Roche, Sanofi-Genzyme, Karl Skriner: None declared DOI: 10.1136/annrheumdis-2019-eular.4653

\section{SAT0050 TNF-A INDUCES MITOPHAGY FORMATION IN RHEUMATOID ARTHRITIS SYNOVIAL FIBROBLAST AND INHIBITION OF MITOPHAGY AMELIORATES SYNOVITIS IN COLLAGEN ANTIBODY-INDUCED ARTHRITIS}

sang youn jung. Bundang CHA medical Center, Department of Rheumatology, Seongnam, Gyeonggi-do, Korea, Rep. of (South Korea)

Background: Rheumatoid arthritis (RA) is a systemic and chronic autoimmune disease that primarily targets synovial membranes. Mitophagy is a selective form of autophagy which removes damaged mitochondria. When mitochondria become damaged, PINK1 (PTEN-induced putative kinase 1) is accumulated on the outer membrane of damaged mitochondria and induces mitophagy. The study of mitophagy in lung fibrosis and parkinson's diseases has recently been described. However, the role of mitophagy in RA has not been reported yet.

Objectives: The aim of this study was to determine the roles of mitophagy in rheumatoid arthritis synovial fibroblast (RASF) and collagen antibody-induced arthritis (CAIA) mice model.

Methods: We studied the mitophagy marker, PINK1, in RASF treated with TNF-a by western blotting and immunofluorescence. Arthritis was induced in PINK1 knockout mice by intraperitoneal (i.p.) injection of antitype II collagen antibody cocktail, followed by i.p injection of lipopolysaccharide. The severity of rheumatoid arthritis was histopathologically assessed

Results: PINK1 expression and damaged mitochondria increased in RASF treated with TNF- $\alpha$. TNF- $\alpha$ produced Intracellular ROS in RASF and mitophagy was regulated by ROS. PINK1 knockdown RASF decreased migration and invasion function. Histopathological examination revealed that the paws from PINK1-knockout mice showed markedly reduced swelling and inflammation compared with those of wild type mice

Conclusion: Taken together, the results suggest that regulation of PINK1 expression in RA may represent potential therapeutic and diagnostic targets for RA
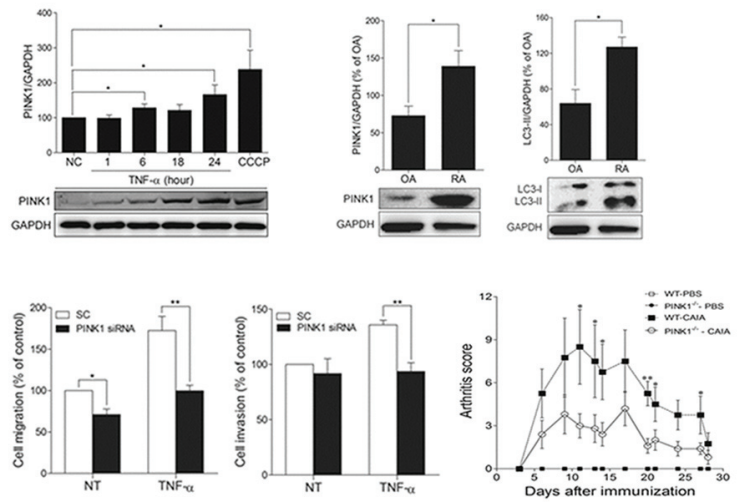

\section{REFERENCES}

[1] Huber LC, Distler O, Tarner I, Gay RE, Gay S, Pap T. Synovial fibroblasts: key players in rheumatoid arthritis. Rheumatology (Oxford). 2006;45 (6):669-75 